\title{
Erratum to: Skin Microstructure is a Key Contributor to Its Friction Behaviour
}

\author{
Maria F. Leyva-Mendivil ${ }^{1,2}$ (I) Jakub Lengiewicz ${ }^{3} \cdot$ Anton Page $^{4} \cdot$ Neil W. Bressloff $^{5}$. $^{-}$ \\ Georges Limbert ${ }^{1,2,6}$
}

Published online: 18 September 2017

(C) Springer Science+Business Media, LLC 2017

\section{Erratum to: Tribol Lett (2017) 65:12 DOI 10.1007/s11249-016-0794-4}

The original version of this article unfortunately contained a mistake. The presentation of Equation (6) was incorrect in the original version of this article.
The denominator corresponding to the described $\kappa_{0}$ contains the coefficient " 4 " that should be " 6 ".

The corrected equation is given below.

$c_{10}=\frac{E}{4(1+v)}$ and $\kappa_{0}=\frac{E}{6(1-2 v)}$

The online version of the original article can be found under doi:10.1007/s11249-016-0794-4.

Georges Limbert

g.limbert@soton.ac.uk

1 National Centre for Advanced Tribology at Southampton (nCATS), Faculty of Engineering and the Environment, University of Southampton, Southampton SO17 1BJ, UK

2 Bioengineering Science Group, Faculty of Engineering and the Environment, University of Southampton,

Southampton SO17 1BJ, UK

3 Institute of Fundamental Technological Research, Polish Academy of Sciences (IPPT PAN), ul. Pawinskiego 5B, 02-106 Warsaw, Poland

4 Biomedical Imaging Unit, Faculty of Medicine, University of Southampton, Southampton General Hospital, Southampton SO16 6YDJ, UK

5 Computational Engineering and Design Group, Faculty of Engineering and the Environment, University of Southampton, Southampton SO17 1BJ, UK

6 Laboratory of Biomechanics and Mechanobiology, Division of Biomedical Engineering, Department of Human Biology, Faculty of Health Sciences, University of Cape Town, Observatory, Cape Town 7935, South Africa 